\title{
Modeling and Compensation for Hysteresis Nonlinearity of a Piezoelectrically Actuated Fast Tool Servo Based on a Novel Linear Model
}

\author{
Zhiwei Zhu, ${ }^{1}$ Xiaoqin Zhou, ${ }^{1}$ Jieqiong Lin, ${ }^{2}$ and Qiang Liu ${ }^{1}$ \\ ${ }^{1}$ School of Mechanical Science and Engineering, Jilin University, Changchun 130022, China \\ ${ }^{2}$ School of Electromechanical Engineering, Changchun University of Technology, Changchun 130012, China \\ Correspondence should be addressed to Xiaoqin Zhou, xqzhou@jlu.edu.cn
}

Received 5 September 2012; Accepted 21 September 2012

Academic Editors: J. Clayton, K. Ismail, and J.-I. Jang

Copyright (C) 2012 Zhiwei Zhu et al. This is an open access article distributed under the Creative Commons Attribution License, which permits unrestricted use, distribution, and reproduction in any medium, provided the original work is properly cited.

\begin{abstract}
In order to describe and compensate for complex hysteresis nonlinearities of piezoelectrically actuated fast tool servo (FTS), a novel Linear Fractional order Differentiation Hysteresis ( $L F D H)$ model is proposed in this paper. By means of the proposed LFDH model which is established on the fractional calculus theory, an analytical description of hysteresis behaviors of the FTS is derived. Furthermore, the LFDH model-based inverse compensation strategy is proposed to suppress the hysteresis effects of the FTS. Finally, a series of experiments are conducted to verify the effectiveness of the LFDH model and the corresponding compensation approach. The results demonstrate that the proposed LFDH model is efficient for describing hysteresis behaviors and the inverse compensation strategy can significantly suppress the inherent hysteresis of the FTS in open-loop operations.
\end{abstract}

\section{Introduction}

Fast-tool-servo-(FTS-) based single-point diamond turning (SPDT) is considered a very promising technology for the generation of freeform surfaces and complicated micro/nano structures [1-3], and the piezoelectric actuator (PEA) has been widely employed as the driving source of the FTS for its various superior advantages, such as high stiffness, high-frequency response, high resolution, and miniature size [1-4]. However, due to the intrinsic friction among the material crystals of the PEA, there always exists nonlinear hysteresis effects when electric voltages are applied to drive the PEA $[4,5]$. Apparently, the hysteresis nonlinearity would significantly limit the positioning performance of the cutting tool of the FTS and even lead to the instability of the servo system, consequently limiting developments of FTS-based micro/nano machining [5-7]. To overcome these limitations, accurate models for hysteresis behaviors should be critically constructed to accordingly develop compensation strategies or enhance the performance of designed controllers.

Motivated by this, extensive mathematical models for hysteresis have been developed, and they may be classified into two aspects: physics-based [5] and phenomenological models [6-8]. The main disadvantage of physics-based models is that they require a large number of materialrelated parameters. The phenomenological models are more relatively suitable and have found widespread acceptance for practical applications in engineering. Various types of such models have been introduced, such as the Preisach model [6, 9], the Prandtl-Ishlinskii (PI) model [10, 11], the Bouc-Wen differential model [12], the turning voltage-based model $[7,13]$, and the neural network-based intelligent model $[14,15]$. Essentially, almost all of these currently developed models are based on complex nonlinearity operators. The main disadvantages of these models can be summarized as follows: (a) these nonlinear operators have significantly limited the implementations of the well-developed analysis and control theories of linear systems to the FTS; (b) these nonlinear operators will add difficulties to the calculation of model inversions and even lead to ill solutions.

Fractional order calculus (FOC) theory, which is a generalization of the conventional integration and differentiation to noninteger orders, has found rapidly increasing applications in various fields $[16,17]$. It has been widely 
believed that FOC could be used to describe a real process more accurately and more flexibly than classical methods [16]. A typical implementation of FOC is the description of dynamic properties of viscoelastic materials [18, 19]. Motivated by these fractional order models for viscoelastic materials, Sunny et al. [20] proposed two models to describe the resistance-strain hysteresis behavior of a conductive polymer sample by combining a series of fractional/integer order functions [20]. Both of the two developed models contained too many parameters to be identified, and the existing hysteresis phenomenon was different from that of PEA.

The present paper aims at presenting a linear scheme for describing hysteresis nonlinearities of piezoelectrically actuated FTS. By taking advantage of the nonlocal memory properties of FOC operations, the nonlocal memorydominant nature of hysteresis nonlinearity is described by a fractional order differential equation (FODE), and a novel Linear Fractional order Differentiation Hysteresis (LFDH) model is accordingly proposed in this paper. In Section 2, the basic definitions and characteristics of FOC theory are introduced. The modeling procedure of the LFDH model and the corresponding inverse compensation strategy are further presented in Section 3. Section 4 focuses on the conduction of experiments to verify the effectiveness of the proposed model and the compensation approach. Finally, the main conclusions of this paper are drawn in Section 5.

\section{A Preliminary to FOC}

Fractional order calculus is a generalization of the conventional integration and differentiation to noninteger orders with the fundamental operator ${ }_{t_{0}} D_{t}^{\alpha} f(t)$, which is defined as

$$
{ }_{t_{0}} D_{t}^{\alpha} f(t)= \begin{cases}\frac{d^{\alpha}}{d t^{\alpha}}, & \operatorname{Re}(\alpha)>0 \\ 1, & \operatorname{Re}(\alpha)=0 \\ \int_{t_{0}}^{t}(d \tau)^{\alpha}, & \operatorname{Re}(\alpha)<0,\end{cases}
$$

where $t_{0}$ and $t$ are the limits of the operation, respectively; $\alpha$ is the order, and $\alpha \in \mathrm{R}$, but $\alpha$ could also be a complex number.

Generally, there exist several well-known definitions of FOC operations including the Grunwald-Letnikov (G-L) definition, the Riemann-Liouville (R-L) definition, and the Caputo definition $[16,21]$. In this paper, the G-L definition is employed to directly carry out the numerical computation of fractional order operations, and it can be expressed as $[16,21]$

$$
\begin{aligned}
t_{0} D_{t}^{\alpha} f(t)= & \lim _{h \rightarrow 0} h^{-\alpha} \sum_{j=0}^{\left[\left(t-t_{0} / h\right)\right]}(-1)^{j} \frac{\Gamma(\alpha+1)}{\Gamma(\alpha-j+1) \Gamma(j+1)} \\
& \times f(t-j h),
\end{aligned}
$$

where $\Gamma(\cdot)$ is the Gamma function, $h$ is the calculation step.

As given in (2), if $f(t)$ is an analytical function of $t$, its fractional derivative ${ }_{0} D_{t}^{\alpha} f(t)$ is an analytical function of $t$

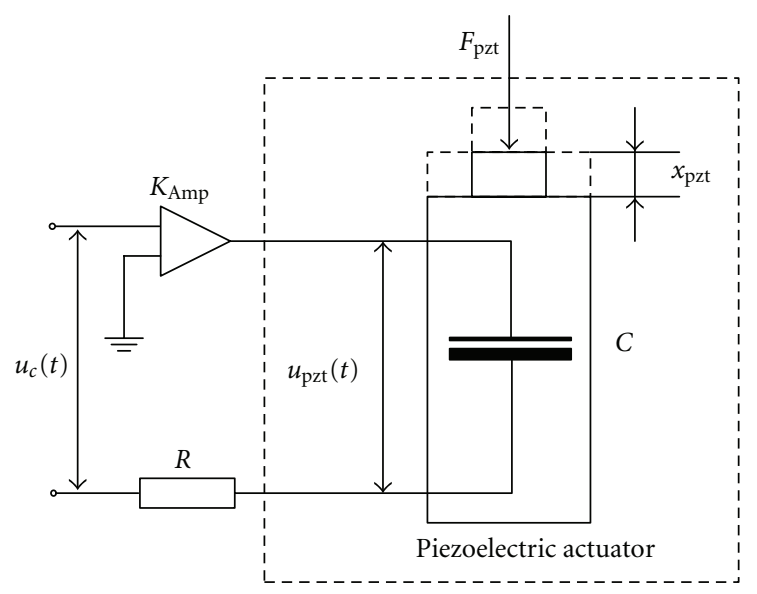

Figure 1: Equivalent driving circuit of piezoelectric actuator.

and $\alpha$, this may increase the flexibility of FOC for representing the modeled objects. Meanwhile, it is evident that the weights of $f(t-j h)$ would decrease with the increase of $j$. It indicates that the fractional order operation possesses nonlocal variable memories with respect to different temporal intervals.

Both, fractional differentiation and integration are linear operations, which satisfy

$$
{ }_{0} D_{t}^{\alpha}[a f(t)+b g(t)]=a \cdot{ }_{0} D_{t}^{\alpha} f(t)+b \cdot{ }_{0} D_{t}^{\alpha} g(t),
$$

where $a$ and $b$ are constant, $g(t)$ is another analytical function of $t$.

For zero initial conditions, the Laplace transform of G-L definition can be written as [16]

$$
L\left[{ }_{0} D_{t}^{\alpha} f(t)\right]=s^{\alpha} L[f(t)]=s^{\alpha} F(s) .
$$

\section{Hysteresis Modeling and Compensation of the FTS}

3.1. The LFDH Model. From an electrical circuit point of view, the PEA can be considered as a capacitive component, and the electric-driven circuit can be illustrated in Figure 1. The relationship between the actual voltage $u_{\mathrm{pzt}}(t)$ applied to the PEA and the control signal $u_{c}(t)$ can be expressed as follows $[22,23]$ :

$$
R C \frac{d u_{\mathrm{pzt}}(t)}{d t}+u_{\mathrm{pzt}}(t)=K_{\mathrm{Amp}} n d_{33} u_{c}(t),
$$

where $n$ denotes the number of layers of the PEA; $d_{33}$ denotes the piezoelectric constant; $R$ and $C$ are the equivalent resistance and capacitance of the PEA, $K_{\mathrm{Amp}}$ is the nominal amplification factor of the power amplifier, and the value of $\tau=R C$ can be regarded as the time constant of the driving circuit.

Assume that the working frequency of the FTS is much lower than its natural frequency and the dynamic behaviors of the mechanism could be ignored, the relationship between 


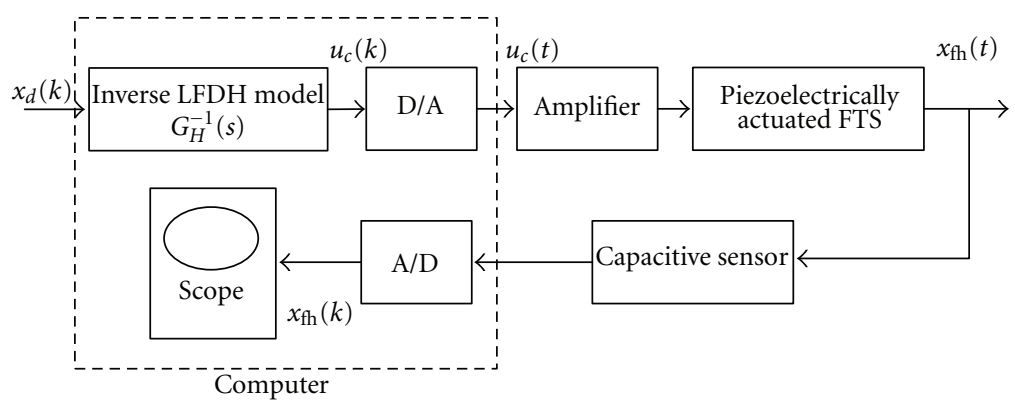

FIgURE 2: Schematic of the LFDH model-based inverse compensation strategy.

the driving force of the PEA and the displacement of the mechanism can be expressed as

$$
\begin{aligned}
F_{\mathrm{pzt}}(t)=k_{\mathrm{pzt}} x_{\mathrm{pzt}} & =n d_{33} k_{\mathrm{pzt}} u_{\mathrm{pzt}}(t) \\
x_{\mathrm{pzt}} & =x_{\mathrm{fh}},
\end{aligned}
$$

where $x_{\mathrm{fh}}$ denotes the displacement of the FTS mechanism, $k_{\mathrm{pzt}}$ and $x_{\mathrm{pzt}}$ denote the equivalent stiffness and the displacement of the PEA, respectively.

Substituting (6) into (5), then the governing equation between the control signal and the corresponding displacement of the mechanism can be obtained as

$$
\frac{R C}{n d_{33}}\left[\frac{d x_{\mathrm{fh}}}{d t}+x_{\mathrm{fh}}(t)\right]=K_{\mathrm{Amp}} n d_{33} u_{c}(t) .
$$

Considering hysteresis effects, we modified (7) based on the FOC theory and proposed a Linear Fractional order Differential Hysteresis (LFDH) model.

By introducing the fractional order differentiation, the governing equation of the FTS can be expressed as

$$
\begin{aligned}
\chi \frac{R C}{n d_{33}}{ }_{0} D_{t}^{\mu} x_{\mathrm{fh}}(t)+\frac{R C}{n d_{33}} x_{\mathrm{fh}}(t) \\
=\rho K_{\mathrm{Amp}} n d_{33}\left[u_{c}(t)+\kappa_{0} D_{t}^{\delta} u_{c}(t)\right] \\
0<\mu<1, \quad 0<\lambda<1, \quad \chi, \rho, \kappa, \mu, \delta \in \mathbb{R},
\end{aligned}
$$

where $\chi, \rho, \kappa$ are constant, $\mu$ and $\delta$ are the differentiation orders of the displacement and the command signal, respectively.

Taking the Laplace transform of two sides in (8) yields

$$
\begin{aligned}
G_{H}(s) & =\frac{X_{\mathrm{fh}}(s)}{U_{c}(s)}=\frac{\rho K_{\mathrm{Amp}}\left(n d_{33}\right)^{2}\left(1+\kappa s^{\delta}\right)}{R C\left(1+\chi s^{\mu}\right)}, \\
0 & <\mu<1, \quad 0<\lambda<1, \quad \chi, \rho, \kappa, \mu, \delta \in \mathbb{R},
\end{aligned}
$$

where $s$ represents the Laplace operator, $G_{H}(s)$ represents the transfer function of the FTS mechanism considering its hysteresis nonlinearity, and $X_{\mathrm{fh}}(s)$ and $U_{c}(s)$ denote the Laplace transform of $x_{\mathrm{fh}}(t)$ and $u_{c}(t)$, respectively.

3.2. The LFDH-Based Inverse Compensation Strategy. The inverse compensation for hysteresis effects of the piezoelectrically actuated FTS relies on the capacity of hysteresis predictions of the LFDH model. Figure 2 illustrates the principle of the compensation strategy in open-loop operations. The main characteristic of the strategy is that the control signal would primarily be compensated by the inverse model and then applied to the PEA.

As for compensation operations, it is essential to obtain the compensated control signal $u_{c}(k)$ corresponding to the desired displacement $x_{d}(k)$. As for conventional hysteresis models, the model inversions are difficult to be achieved due to their complex nonlinear operators $[6,10,11,13]$. However, by taking advantage of the linear frame of the LFDH model in this paper, the analytical description of the inverse LFDH model can be directly accessed as

$$
\begin{gathered}
G_{H}^{-1}(s)=\frac{U_{c}(s)}{X_{\mathrm{fh}}(s)}=\frac{R C\left(1+\chi s^{\mu}\right)}{\rho K_{\mathrm{Amp}}\left(n d_{33}\right)^{2}\left(1+\kappa s^{\delta}\right)}, \\
0<\mu<1, \quad 0<\lambda<1, \quad \chi, \rho \kappa, \mu, \delta \in \mathbb{R} .
\end{gathered}
$$

Generally, to realize the LFDH model-based inverse compensation, there exist three main steps, namely, modeling and identification, compensated command signal calculation, and application. These steps could be formalized as follows.

Step 1 (modeling and identification). In this stage, a set of study signals are specified to obtain the characteristics of the FTS system, and then the parameters of the LFDH model are estimated based on certain evolutionary optimization schemes (EOS).

Step 2 (compensated command signal calculation). In this stage, a set of desired displacement values $x_{d}(k)$ are created, and then the compensated control signal $u_{c}(k)$ is calculated according to the inverse LFDH model as shown in (10).

Step 3 (application). In this stage, the compensated control signal is applied to drive the FTS to achieve the desired displacements.

\section{Experiment Results}

4.1. Experiment Setup. The authors of this paper carefully designed a short stroke FTS for ultraprecision diamond turning in [4], where the cutting tool is actuated by the PEA and guided by flexure hinges. The position and dimension parameters of this FTS mechanism were determined by a multi-objective optimum approach. It could reach up to 


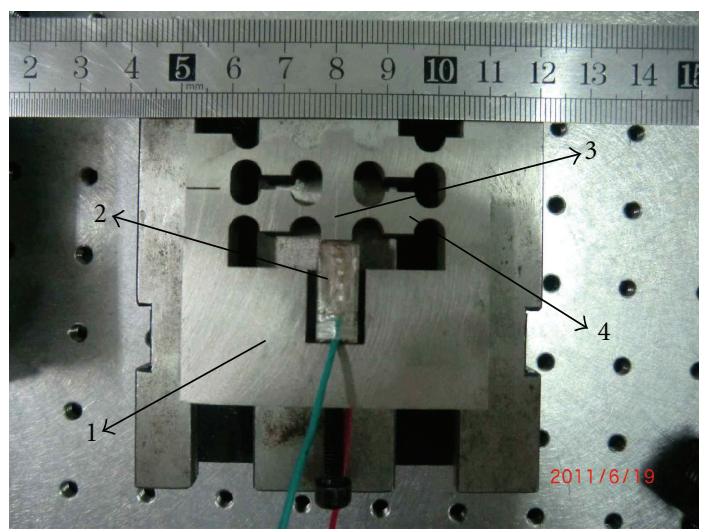

FIgUre 3: Photographic of the FTS mechanism ((1) the base; (2) the piezoelectric actuator; (3) the tool holder; (4) the flexure hinges).

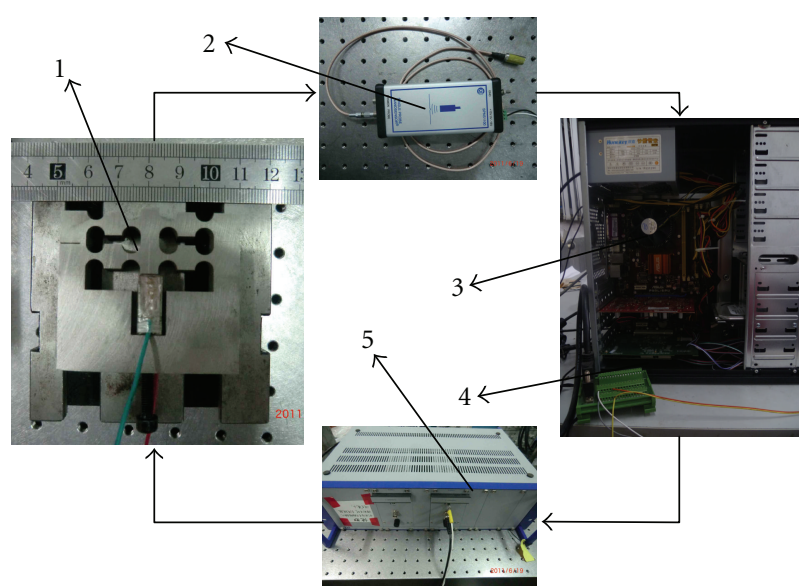

FIgURE 4: Schematic of the experimental system ((1) the FTS mechanism; (2) the capacitive transducer; (3) the computer equipped with data-acquisition card; (4) the data-acquisition card from ADLINK; (5) the power amplifier module PI E-617).

a stroke of $10.25 \mu \mathrm{m}$ with at least $2 \mathrm{kHz}$ bandwidth, and the hysteresis phenomenon is about $19.5 \%$ of the stroke. The photographic of the designed FTS is presented in Figure 3. This FTS mechanism consists of four main parts, namely, the base, the PEA, the tool holder, and the flexure hinges. Under working conditions, the tool holder is actuated by the PEA and guided by a group of parallel and symmetric hinges, which are designed as circular notch-type hinges.

Figure 4 illustrates the test and measurement equipment of the experiment part. As shown in Figure 4, a computer is used to generate the command signals for the PEA and implement the control procedure in Section 4.3. The generated signal is converted through a data-acquisition card from ADLINK and amplified by an amplifier module PI E-617 with a nominal amplification factor $10 \pm 0.1$. Capacity transducer-based sensing methodology, which is frequently employed for trajectory tracking of FTS, is chosen for dynamic position measurement. The resolution of the high precision capacitive sensor is $0.0077 \%$ of the full stroke, and its working bandwidth is up to $35 \mathrm{kHz}$, with an effective measurement range of $200 \mu \mathrm{m}$. The measured signal is converted to a digital signal by the data-acquisition card, and then gathered and stored in the computer for further analysis. To reduce the external disturbances, the experiments are carried out on a vibration-isolated airbearing platform.

4.2. Identification of the LFDH Model. For the identification process, a hybrid signal with variable amplitudes is employed as the command signal, and the corresponding output of the FTS mechanism is measured via the high precision capacitive sensor. The frequency of the command signal is especially chosen as $1 \mathrm{~Hz}$. The low frequency chosen here can avoid possible separations between the PEA and the tool holder during rapid expansions and retractions of the PEA [6], and the hypothesis that the displacement of the PEA is equivalent to that of the mechanism can come into existence.

Similar EOSs as shown in $[24,25]$ are established for the estimation of parameters of the LFDH model. The meansquare-error (MSE) is employed as the objective function, and an improved differential evolution (IDE) algorithm is utilized as the searching tool for the optimal parameters. The identification procedure will be detailed in our future work.

Accordingly, the identified LFDH model can be obtained as

$$
G_{H}(s)=\frac{X(s)}{U_{c}(s)}=\frac{11.3885 s^{0.046153}+1}{16.523 s^{0.44346}+79.6379}
$$

The displacements generated by the FTS and the identified model are illustrated in Figure 5(a), the hysteresis loop generated by the FTS and the model in several periods are presented in Figure 5(b), and the modeling error is illustrated in Figure 5(c). As shown in Figures 5(a) and 5(b), the response generated by the proposed model agrees well with the actual response measured from the FTS mechanism. From the resultant error shown in Figure 5(c), the maximum modeling error in the steady state is about $\pm 0.15 \mu \mathrm{m}$, which is about $\pm 1.75 \%$ of the full span range. The results demonstrate that the LFDH model could fit experimental data with a good degree of accuracy.

4.3. Inverse Compensation for the Hysteresis. According to the identified LFDH model as presented in (11), the analytical description of the inverse LFDH model can be simply accessed as

$$
G_{I}(s)=G_{H}^{-1}(s)=\frac{16.523 s^{0.44346}+79.6379}{11.3885 s^{0.046153}+1} .
$$

In order to assess the LFDH model-based inverse compensation approach in open-loop operations, a series of experiments are carried out. Three typical signals, which are respectively illustrated in Figures 6(a), 7(a), and 8(a), are employed as the desired trajectories. The preshaped command signals generated by the inverse LFDH model are respectively illustrated in Figures 6(b), 7(b), and 8(b). With these compensated commands, the resultant responses are derived as shown in Figures 6(a), 7(a), and 8(a), and 


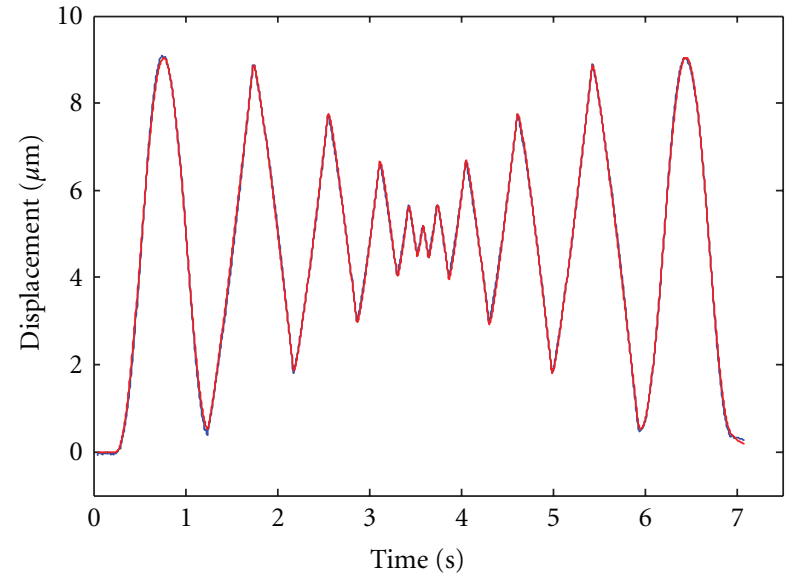

(a) The displacements generated by the FTS and the model

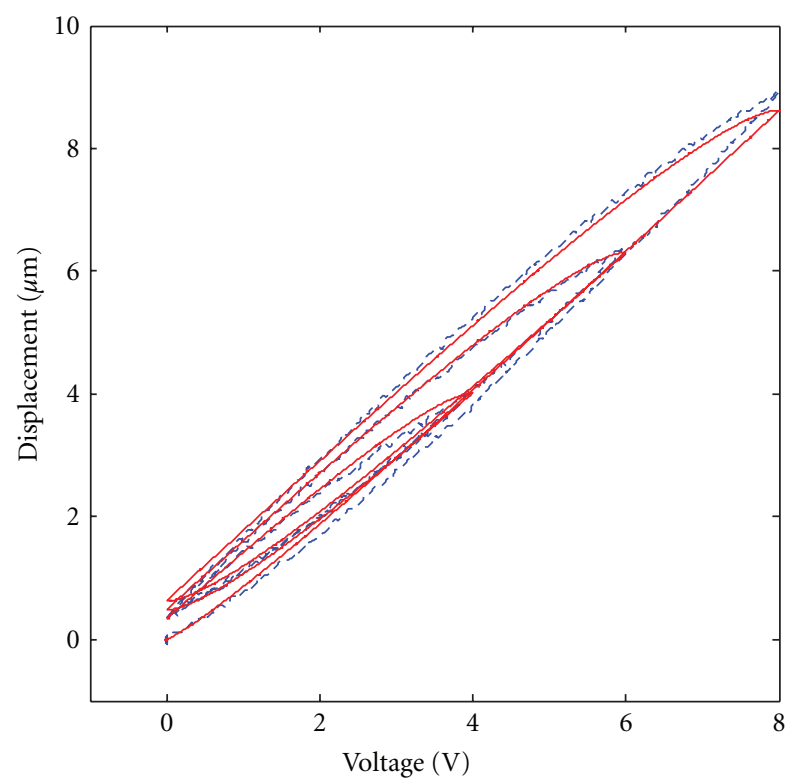

(b) Hysteresis loop generated by the FTS and the model

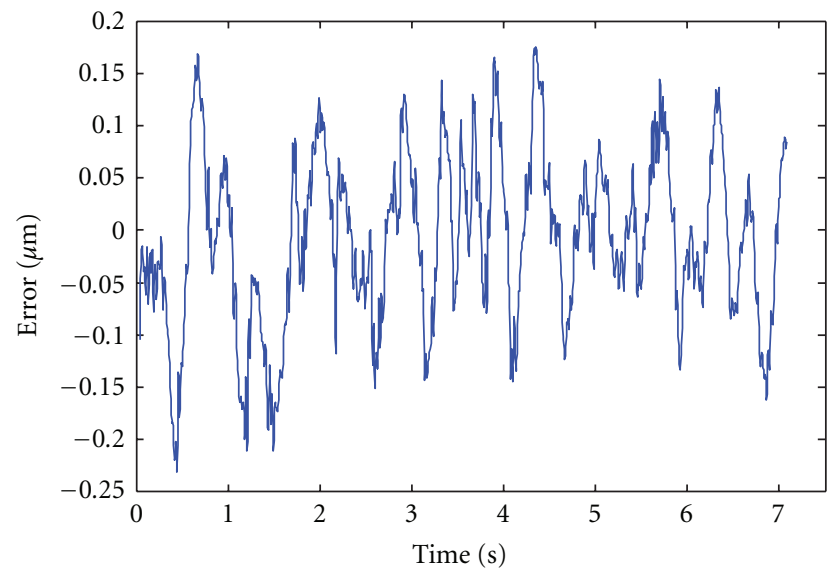

(c) The modeling error

Figure 5: Modeling results. (In (a) and (b), the blue line and the red line denote the displacement generated by the FTS and the LFDH model, resp.).

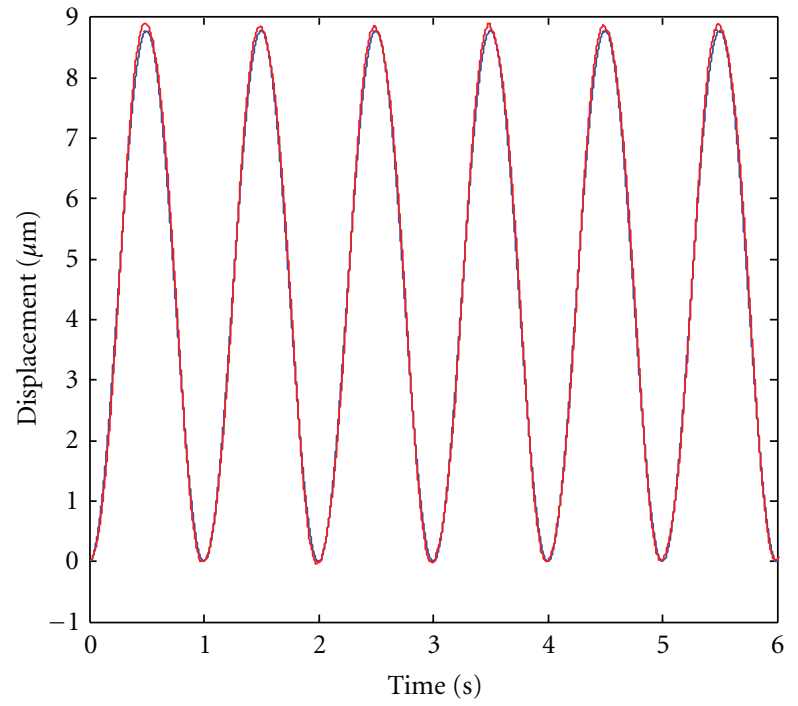

(a) The desired trajectory and the tracking trajectory

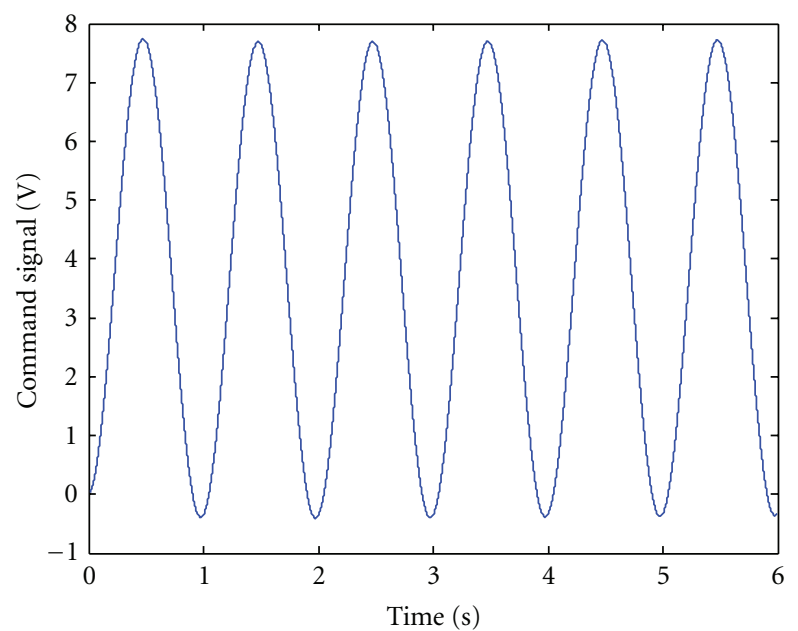

(b) The compensated control signal

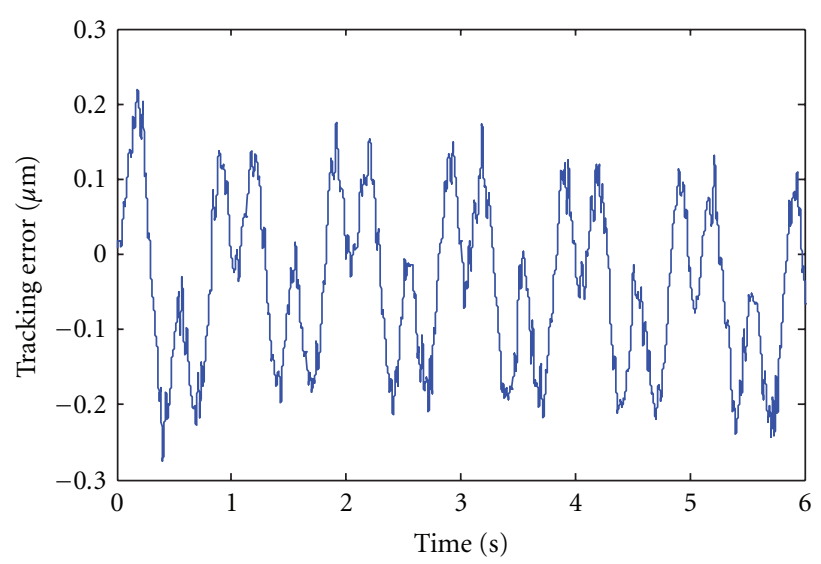

(c) The tracking error

FIGURE 6: Responses of the FTS mechanism with inverse compensation. (In (a), the blue line and the red line denote the desired trajectory and the tracking trajectory, resp.). 


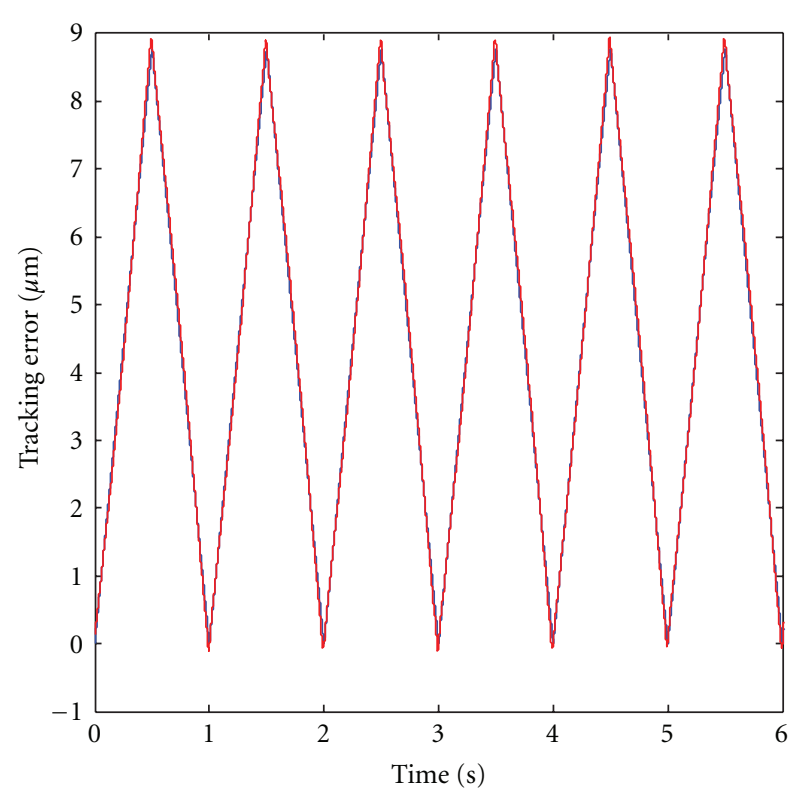

(a) The desired trajectory and the tracking trajectory

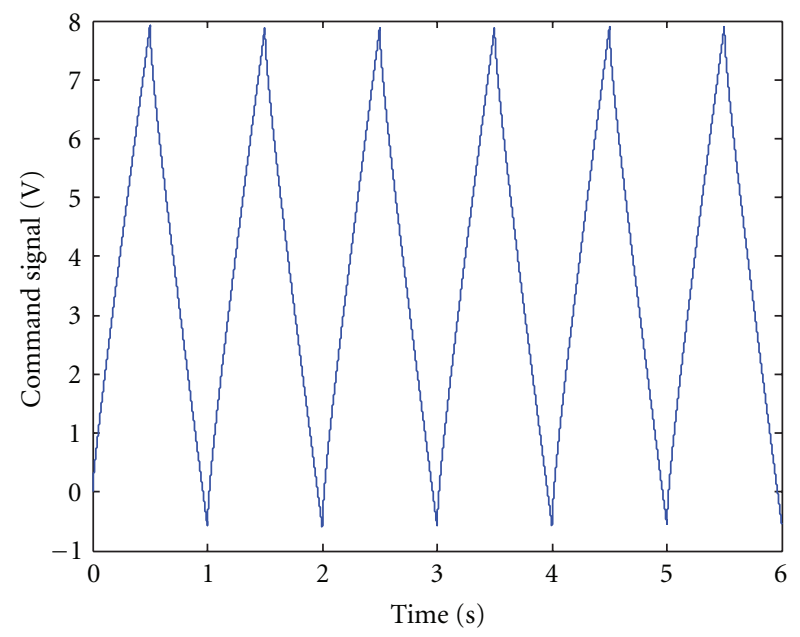

(b) The compensated control signal

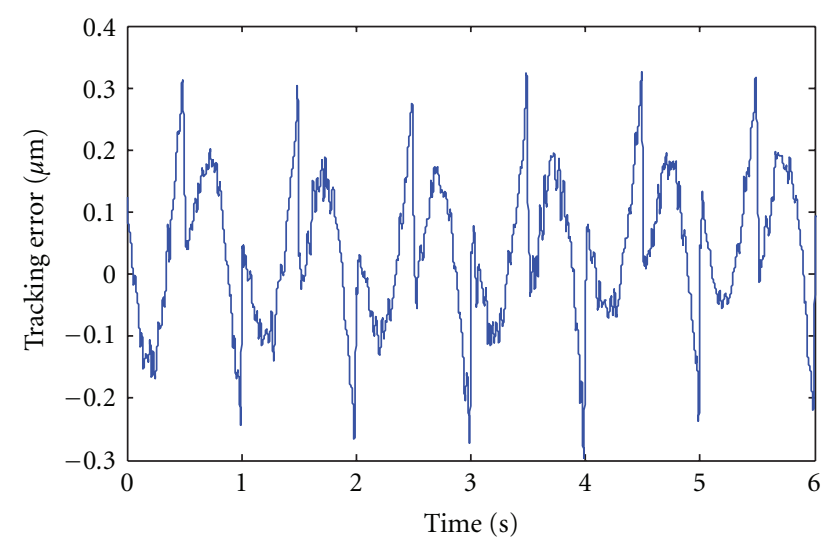

(c) The tracking error

FIGURE 7: Responses of the FTS mechanism with inverse compensation. (In (a), the blue line and the red line denote the desired trajectory and the tracking trajectory, resp.).

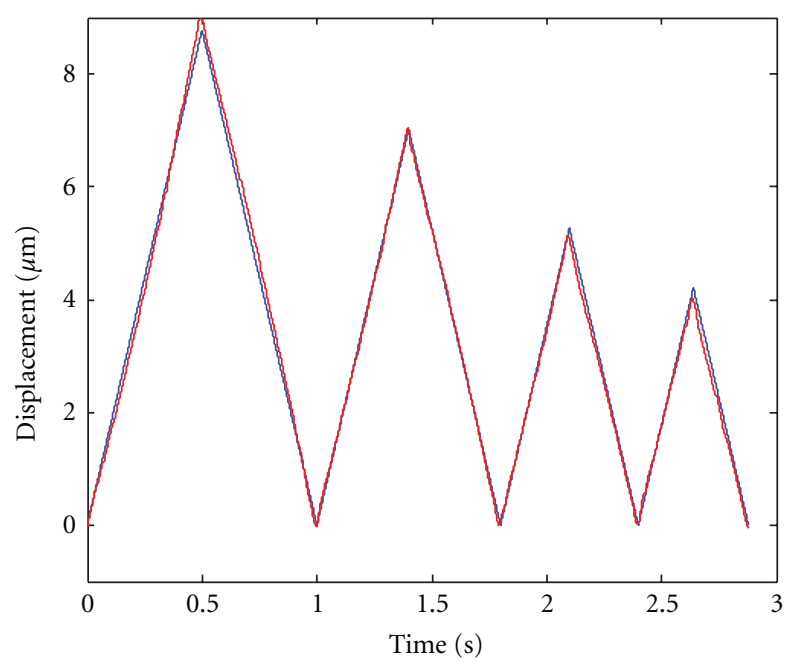

(a) The desired trajectory and the tracking trajectory

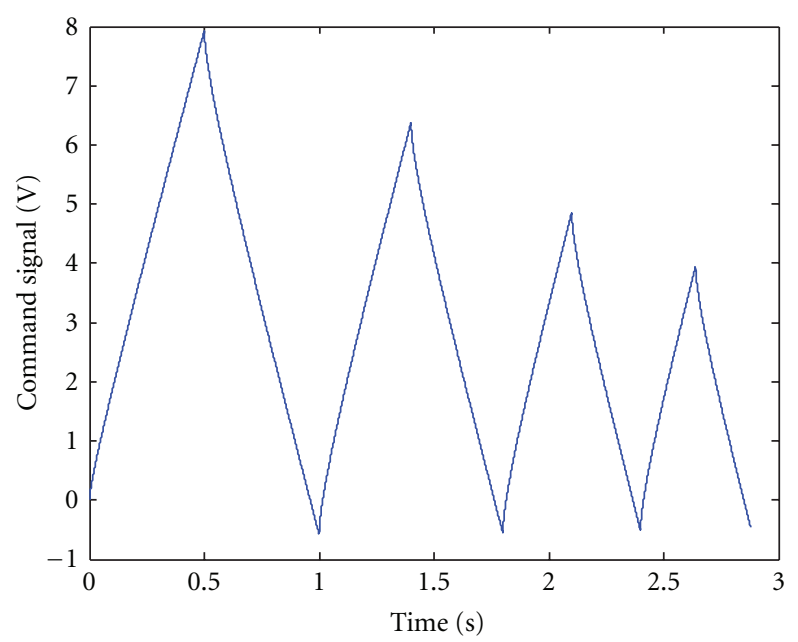

(b) The compensated control signal

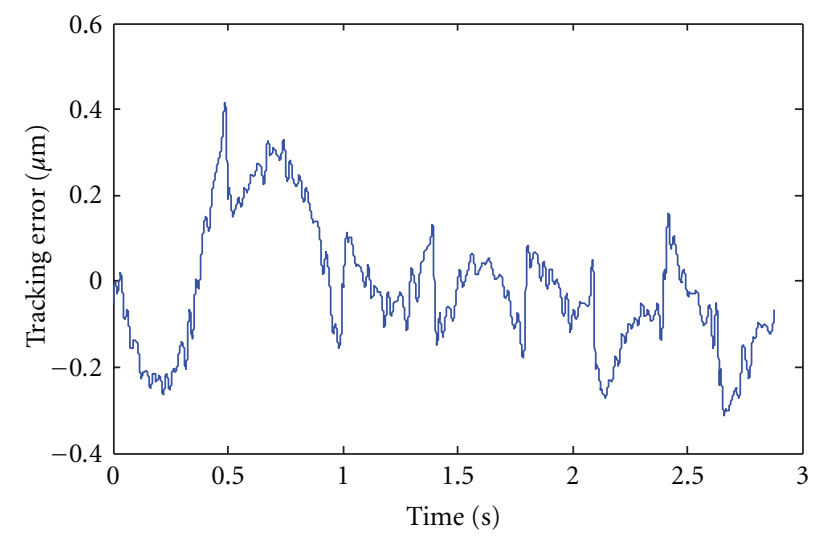

(c) The tracking error

FIGURE 8: Responses of the FTS mechanism with inverse compensation. (In (a), the blue line and the red line denote the desired trajectory and the tracking trajectory, resp.). 
the tracking errors are further shown in Figures 6(c), 7(c), and 8(c). As shown in Figures 6(b), 7(b), and 8(b), to reach the zero outputs with zero inputs, proper negative voltages are applied to the PEA to compensate for the displacement drifts. From the comparisons between the desired outputs and the experiment outputs as illustrated in Figures 6(a), $7(\mathrm{a})$, and $8(\mathrm{a})$, the tracking trajectories agree well with the desired trajectories. And from the resultant errors shown in Figures $6(\mathrm{c}), 7(\mathrm{c})$, and $8(\mathrm{c})$, the tracking errors in the steady state are all less than $\pm 0.2 \mu \mathrm{m}$. It should be noticed that the tracking errors in the initial stage are much larger. This phenomenon may be due to the dynamic characteristics of the FTS mechanism, which is not considered in our current work.

As we have reported before, the relative error of hysteresis nonlinearity of this FTS mechanism is about $19.5 \%$. But after the proposed compensation strategy is implemented, the relative error caused by hysteresis nonlinearity is strongly reduced to less than $\pm 2 \%$. All of the results indicate that the LFDH model-based inverse compensation approach can significantly suppress the inherent hysteresis of the FTS in open-loop operations.

\section{Conclusions}

The main contribution of this paper is to develop a linear mathematical model to describe the complex hysteresis nonlinearity of piezoelectrically actuated FTS. Based on the linear frame, it enables us to implement the welldeveloped analysis and control theories of linear system into FTS system. The proposed model is constructed based on fractional calculus theory. It can give an analytical description of hysteresis. To verify the effectiveness of the LFDH model and the inverse compensation approach, a series of experiments are conducted. The maximum modeling error is about $\pm 1.75 \%$ of the full span range, and the relative positioning error can be strongly reduced to less than $\pm 2 \%$ by implementing the inverse compensation approach. The results verify that the proposed LFDH model is efficient for describing the hysteresis behaviors and that the LFDH model-based inverse compensation approach could significantly suppress inherent hysteresis effects in open-loop operations.

\section{Acknowledgments}

The authors are grateful to the financial support from the NSF of China (51175221; 51075041; 50995077), the Ministry of Science and Technology (MoSt) of China (2008AA04Z125), the Ministry of Education (MoE) of China (20070183104), the Department of Science \& Technology (DoST) of Jilin Province, China (20090337; 20100359), and the Youth Technology Foundation (YTF) of Jilin University.

\section{References}

[1] Z. Zhu, X. Zhou, Q. Liu, J. Lin, and S. Zhao, "Fabrication of micro-structured surfaces on bulk metallic glasses based on fast tool servo assisted diamond turning," Science of Advanced Materials, vol. 4, no. 9-10, 2012.

[2] W. Gao, T. Araki, S. Kiyono, Y. Okazaki, and M. Yamanaka, "Precision nano-fabrication and evaluation of a large area sinusoidal grid surface for a surface encoder," Precision Engineering, vol. 27, no. 3, pp. 289-298, 2003.

[3] H. S. Kim, K. I. Lee, K. M. Lee, and Y. B. Bang, "Fabrication of free-form surfaces using a long-stroke fast tool servo and corrective figuring with on-machine measurement," International Journal of Machine Tools and Manufacture, vol. 49, no. 12-13, pp. 991-997, 2009.

[4] Z. Zhu, X. Zhou, Q. Liu, and S. Zhao, "Multi-objective optimum design of fast tool servo based on improved differential evolution algorithm," Journal of Mechanical Science and Technology, vol. 25, no. 12, pp. 3141-3149, 2011.

[5] T. J. Yeh, H. Ruo-Feng, and L. Shin-Wen, "An integrated physical model that characterizes creep and hysteresis in piezoelectric actuators," Simulation Modelling Practice and Theory, vol. 16, no. 1, pp. 93-110, 2008.

[6] Y. Ting, C. C. Li, and C. M. Lin, "Controller design for highfrequency cutting using a piezo-driven microstage," Precision Engineering, vol. 35, no. 3, pp. 455-463, 2011.

[7] C. Ru and L. Sun, "Improving positioning accuracy of piezoelectric actuators by feedforward hysteresis compensation based on a new mathematical model," Review of Scientific Instruments, vol. 76, no. 9, Article ID 095111, 8 pages, 2005.

[8] S. Bashash and N. Jalili, "Underlying memory-dominant nature of hysteresis in piezoelectric materials," Journal of Applied Physics, vol. 100, no. 1, Article ID 014103, 6 pages, 2006.

[9] S. Mittal and C. H. Menq, "Hysteresis compensation in electromagnetic actuators through Preisach model inversion," IEEE/ASME Transactions on Mechatronics, vol. 5, no. 4, pp. 394-409, 2000.

[10] U. X. Tan, W. T. Latt, C. Y. Shee, C. N. Riviere, and W. T. Ang, "Feedforward controller of ill-conditioned hysteresis using singularity-free Prandtl-Ishlinskii model," IEEE/ASME Transactions on Mechatronics, vol. 14, no. 5, pp. 598-605, 2009.

[11] U. X. Tan, W. T. Latt, F. Widjaja, C. Y. Shee, C. N. Riviere, and W. T. Ang, "Tracking control of hysteretic piezoelectric actuator using adaptive rate-dependent controller," Sensors and Actuators A, vol. 150, no. 1, pp. 116-123, 2009.

[12] M. Ye and X. Wang, "Parameter estimation of the Bouc-Wen hysteresis model using particle swarm optimization," Smart Materials and Structures, vol. 16, no. 6, pp. 2341-2349, 2007.

[13] L. Sun, C. Ru, W. Rong, L. Chen, and M. Kong, "Tracking control of piezoelectric actuator based on a new mathematical model," Journal of Micromechanics and Microengineering, vol. 14, no. 11, pp. 1439-1444, 2004.

[14] X. Dang and Y. Tan, "An inner product-based dynamic neural network hysteresis model for piezoceramic actuators," Sensors and Actuators A, vol. 121, no. 2, pp. 535-542, 2005.

[15] W. Guo D Liu Wang, "Neural network hysteresis modeling with an improved preisach model for piezoelectric actuator," Engineering Computations, vol. 29, no. 3, pp. 248-259, 2012.

[16] Y. Q. Chen, I. Petráš, and D. Xue, "Fractional order control-a tutorial," in 2009 American Control Conference (ACC '09), pp. 1397-1411, St. Louis, Mo, USA, June 2009.

[17] J. T. Machado, V. Kiryakova, and F. Mainardi, "Recent history of fractional calculus," Communications in Nonlinear Science and Numerical Simulation, vol. 16, no. 3, pp. 1140-1153, 2011.

[18] J. J. d Espi'ndol, J. M. da Silva Neto, and E. M. O. Lopes, "A generalized fractional derivative approach to viscoelastic 
material properties measurement.," Applied Mathematics and Computation, vol. 164, no. 2, pp. 493-506, 2005.

[19] F. C. Meral, T. J. Royston, and R. Magin, "Fractional calculus in viscoelasticity: an experimental study," Communications in Nonlinear Science and Numerical Simulation, vol. 15, no. 4, pp. 939-945, 2010.

[20] M. R. Sunny, R. K. Kapania, R. D. Moffitt, A. Mishra, and N. Goulbourne, "A modified fractional calculus approach to model hysteresis," Journal of Applied Mechanics, vol. 77, no. 3, Article ID 031004, 8 pages, 2010.

[21] M. S. Tavazoei, "A note on fractional-order derivatives of periodic functions," Automatica, vol. 46, no. 5, pp. 945-948, 2010.

[22] Y. Tian, B. Shirinzadeh, and D. Zhang, "A flexure-based mechanism and control methodology for ultra-precision turning operation," Precision Engineering, vol. 33, no. 2, pp. 160-166, 2009.

[23] Y. Tian, D. Zhang, and B. Shirinzadeh, "Dynamic modelling of a flexure-based mechanism for ultra-precision grinding operation," Precision Engineering, vol. 35, no. 4, pp. 554-565, 2011.

[24] H. Tang, S. Xue, and C. Fan, "Differential evolution strategy for structural system identification," Computers and Structures, vol. 86, no. 21-22, pp. 2004-2012, 2008.

[25] C. C. Kao and R. F. Fung, "Using the modified PSO method to identify a Scott-Russell mechanism actuated by a piezoelectric element," Mechanical Systems and Signal Processing, vol. 23, no. 5, pp. 1652-1661, 2009. 

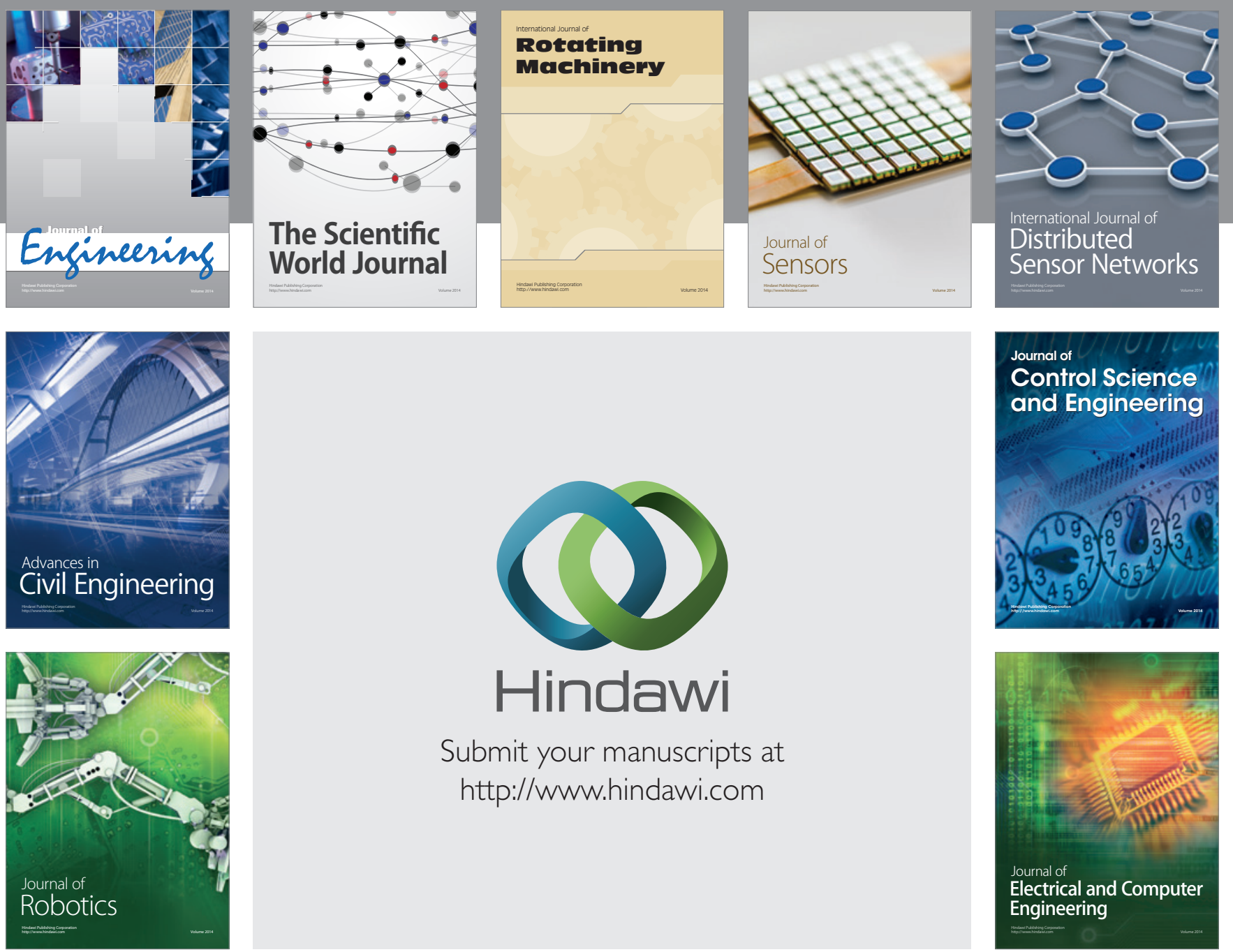

Submit your manuscripts at

http://www.hindawi.com
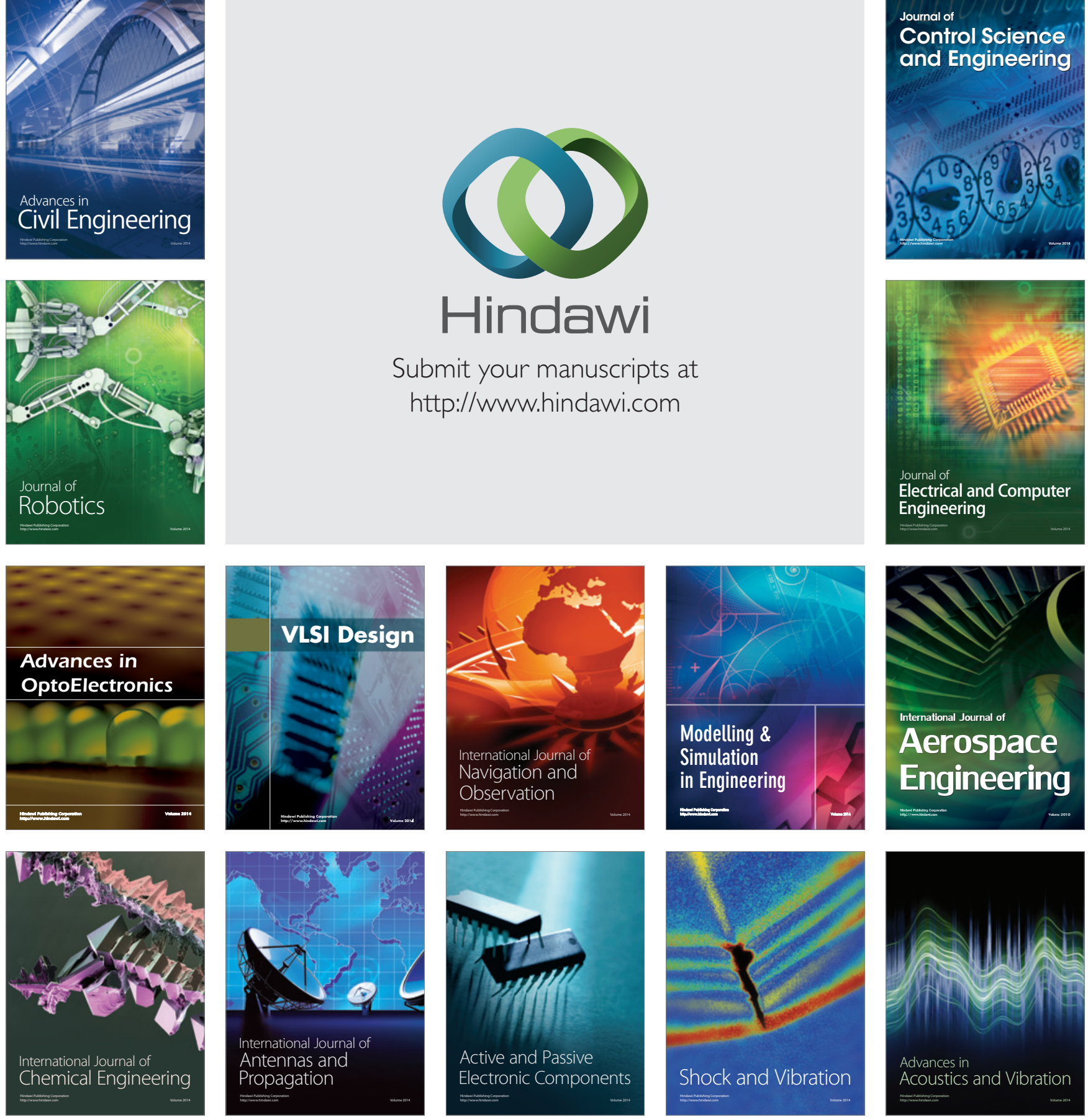